\title{
JUST NOTICEABLE DIFFERENCES FOR VEHICLE RATES OF CLOSURE
}

\author{
Gregory M. Corso and Nicholas J. Kelling \\ Georgia Institute of Technology \\ Atlanta, GA \\ E-mail: gc4@mail.gatech.edu
}

\begin{abstract}
Summary: The goal for this research was to identify the just noticeable difference (JND) for vehicle rates of closure. In our attempt to identify the JND we used two traditional psychophysical methods. However, these procedures resulted aberrant relationships between rate of closure and percent correct. Both of the traditional procedures used a sequential presentation of a standard animation and a comparison animation. The final method used a change in the rate of closure within the animation. This method provided us with a JND of between 12.9 to $16.1 \mathrm{~km} / \mathrm{h}$ ( 8 to $10 \mathrm{mph}$ ). Reasons for the aberrant findings using the traditional methods are discussed.
\end{abstract}

\section{INTRODUCTION}

When following a vehicle, one requirement for the driver of the following vehicle is to detect when the lead vehicle has increased or decreased its speed. Clearly, being able to detect a change in speed of the lead vehicle is necessary in order for the driver of the following vehicle to respond accordingly. The determination in a change of speed of the lead vehicle may not be very important, for example if there is a large distance between the two vehicles. However, in situations where the distance between the two vehicles is very short, the detection of a change in speed of the lead vehicle may be critical. While the initial goal for this research was to identify the just noticeable difference (JND) in rates of closure between two vehicles, it soon became an adventure in identifying a method that would provide us with a means to determine the JND. Traditional psychophysical procedures appeared to be the most appropriate methods for determining the rate of closure JND. It appeared that the method of constant stimuli (Corso, 1967) would not only provide us with a JND value, but the use of this procedure would also provide us with a relationship between rate of closure and percent correctly detected. These data could be used to develop warning systems that would enhance the situational awareness of drivers. However, the method of constant stimuli did not provide us with a percent correct - rate of closure relationship that even approximated the traditional psychophysical relationships usually found.

Not finding a "normal" psychophysical function relating percent correct and rate of closure using the method of constant stimuli, we altered our course and decided to use a modification of the method of limits (Corso, 1967). However, as with the previous method, this method also resulted in the same type of aberrant findings. Hence, we decided to abandon the "traditional" psychophysical methods and simply present a trial where a change might occur. This approach did result in the usual type of psychophysical relations normally found for other types of stimuli. 


\section{METHODS}

\section{Participants}

The first two experiments used six volunteers who were either graduate or undergraduate students at the Georgia Institute of Technology. For the final experiment, nine volunteers from the same pool participated. Only participants with normal or corrected vision were used. All participants were treated according to IRB procedures.

\section{Apparatus}

Participants were tested using an individualized testing station consisting of a PC computer with a $43.2 \mathrm{~cm}$ (17 in) LCD monitor. Only a single participant was run during an experimental session. Participant responses were gathered through the use of the attached keyboard. The order and presentation of each rate of closure was controlled using a program written in Inquisit (Millisecond $^{\mathrm{TM}}$, 2005). Each trial consisted of an animation depicting a road converging in the distance (Carrera ${ }^{\mathrm{TM}} 4$; $\left._{\text {Evia }}{ }^{\mathrm{TM}}, 2005\right)$. At the beginning of each animation, the self-motion appeared as if the participant was inside and driving a vehicle. In the distance, the lead vehicle appeared. The appearance of the lead vehicle was sufficiently distant so the participant was not surprised by its occurrence.

\section{PROCEDURE}

\section{Experiment One}

After granting consent, the participant was seated at the testing apparatus. A constant stimuli method was used for the first procedure. Two sequential animations, a standard and a comparison, were presented. Both animations depict a vehicle traveling toward a parked lead vehicle from the driver's perspective. The participant's task was to determine whether the rate of closure shown in the second animation was the same or different than the rate of closure viewed in the first animation. Each animation was a total of four seconds in duration and stopped before a collision would have occurred. The standard animation could take one of two rates of closure $32.2 \mathrm{~km} / \mathrm{h}(20 \mathrm{mph})$ or $80.5 \mathrm{~km} / \mathrm{h}(50 \mathrm{mph})$. The rate of closure for the comparison animation was randomly chosen and assumed values that started at the standard animations rate and ended $16 \mathrm{~km} / \mathrm{h}(10 \mathrm{mph})$ above this value in $3.2 \mathrm{~km} / \mathrm{h}(2 \mathrm{mph})$ steps. The presentation of the standard animations was counterbalanced. Each participant was exposed to 180 trials for each standard, resulting in 30 data points for each comparison animation.

\section{Experiment Two}

A modification of the method of limits was used for the second experiment. The participant was shown a standard animation, using the same standard animations from the first experiment, followed by a comparison animation. The task of the participant was to decide if there was a difference between the rates of closure shown in the first animation versus that shown in the second animation. If the participant said there was no difference, the rate of closure increased 
and was presented to the participant again, after being shown the standard rate of closure animation. Once the participant decided that a difference between the rates of closure for the two animations existed, higher rates of closure were presented to test the reliability of the choice. The rates of closure of the comparison animations ranged from zero to $32.2 \mathrm{~km} / \mathrm{h}$ (20 mph) above the standard. The duration of each animation was eight seconds. The animation was halted before a collision occurred.

\section{Experiment Three}

A changing stimulus method was utilized for the final procedure. Instead of presenting two sequential animations and requiring the participant to decide if there was a difference in the rate of closure between the two animations, this method used a single continuous animation. In these animations, the lead vehicle remained stopped (closure rate of $32.2 \mathrm{~km} / \mathrm{h}$ ) or immediately increased speed (rates of closure of 0 to $32.2 \mathrm{~km} / \mathrm{h}$ ) within the animation. Eight- $\mathrm{km} / \mathrm{h}$ steps were used across a total range of $32.2 \mathrm{~km} / \mathrm{h}$. The task of the participants was to determine if a change in rate of closure occurred during the animation. Additionally, animations were eight seconds in length with no collision and any speed changes took place five seconds into the animation.

\section{ANALYSIS AND RESULTS}

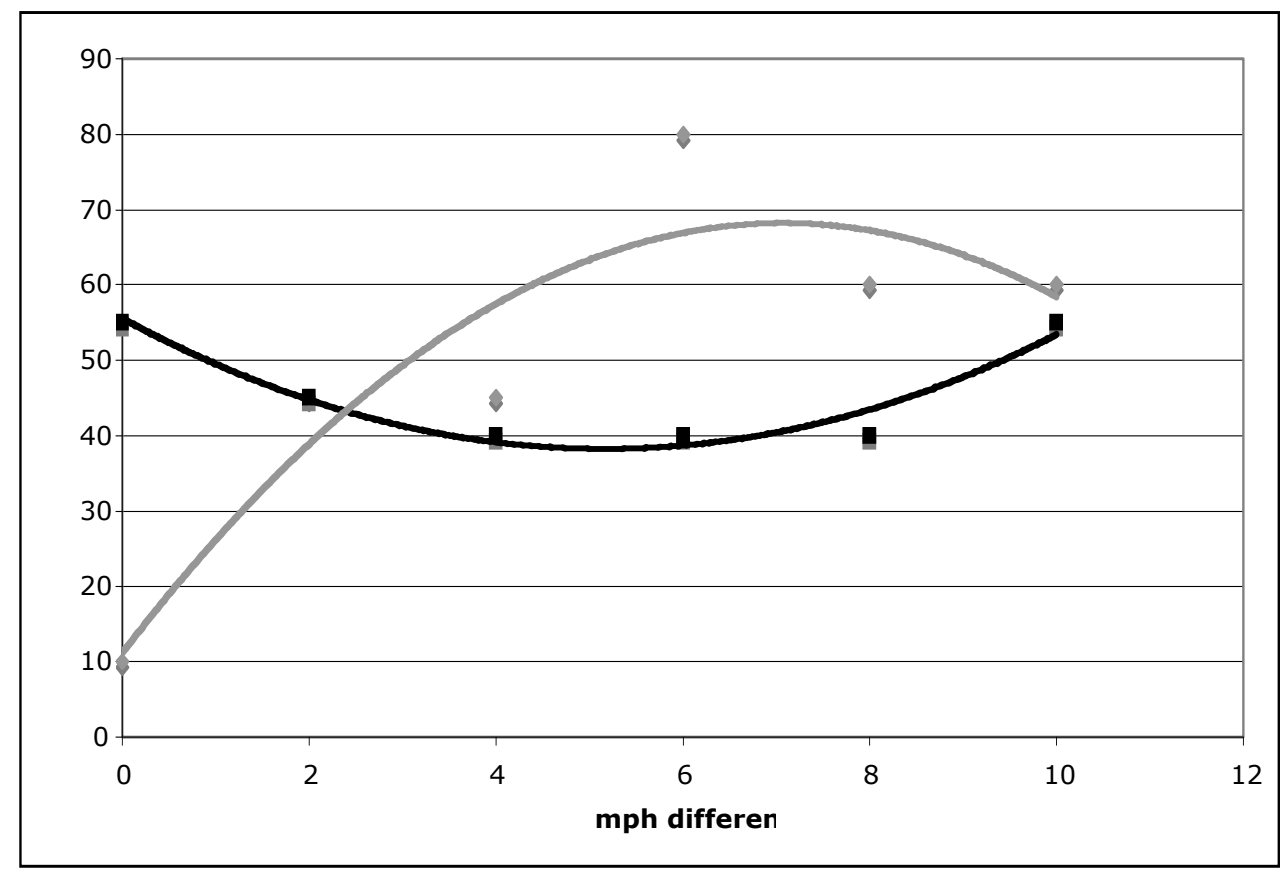

Figure 1. Sample of results from two participants in the $80.5 \mathrm{~km} / \mathrm{h}$ (50 mph) condition of Experiment 1

For each participant and for each procedure, the probability of correctly detecting a difference for each closure rate was plotted and a polynomial function was fit to the data. Sample data from participants are shown in Figures 1 and 2 for Experiments 1 and 2, respectively. These plots depicted large individual differences with very unique relationships between closure rates and 
the probability of detection. In general, non-standard cumulative probability curves were observed for each participant in each method. The changing stimulus procedure, used in Experiment 3, resulted in a much more traditional relationship between the rate of closure and the probability of detection. A representative sample of these plots can be seen in Figure 3 . The generalized finding of Experiment 3 detailed an average JND of between 12.9 to $16.1 \mathrm{~km} / \mathrm{h}$ ( 8 to $10 \mathrm{mph})$ for a change in rate of closure using a $32.2 \mathrm{~km} / \mathrm{h}(20 \mathrm{mph})$ standard.

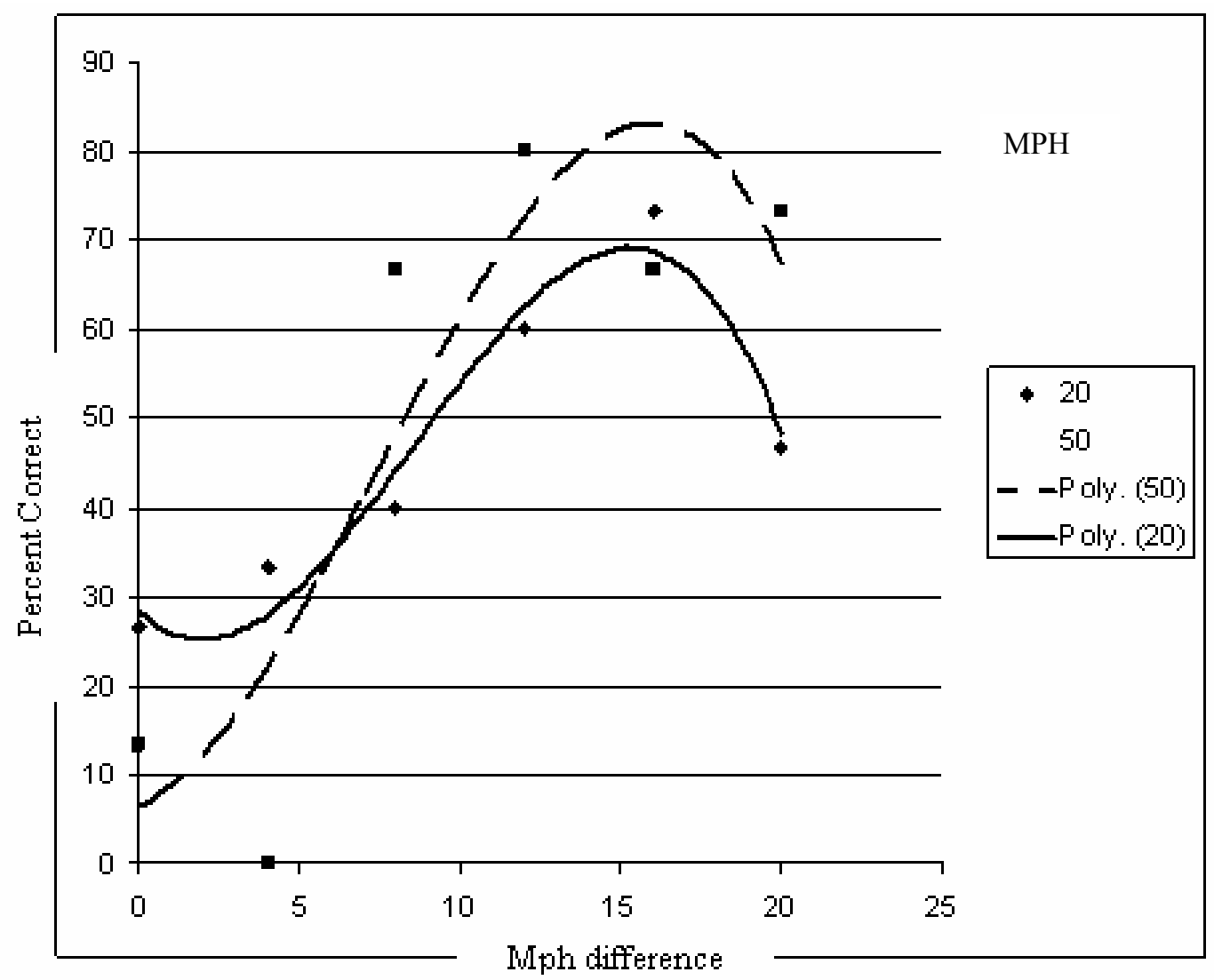

Figure 2. Sample of results from a single participant in experiment 2

\section{DISCUSSION}

As was mentioned above, the changing stimulus method provides us with a JND of $12.9-16.1$ $\mathrm{km} / \mathrm{h}(8-10 \mathrm{mph})$ across the two different base rates of closure. It should also be mentioned that this method may be more ecologically valid than the traditional psychophysical procedures. When driving, we do not have a "no change rate of closure" scenario held in working memory; we rely on bottom-up cues to provide us with information about the change in the rate of closure. What is somewhat alarming is that the JND for the rate of closure is so large. Given that a driver in a lead vehicle can reduce the speed of that vehicle without depressing the brake pedal and that the only cues available to a driver in a following vehicle are those resulting from a change in the rate of closure (including changes in apparent size of the lead vehicle), it would appear that some type of change in rate of closure warning device might be useful. 
Even though the last method provided us with the traditional relationship between rate of closure and percent correct that is usually found for other types of psychophysical phenomena, there are several other issues that could be addressed. The most important of these issues is why the first two methods did not provide us with usable data. One speculation is that the first two procedures relied heavily on working memory and that dynamic stimuli completely exhausted the capacity of working memory. We might also speculate that while it might be possible to hold one dynamic representation in working memory, the presentation of a second dynamic representation either erases the first representation or the perception of the second representation reduces the amount of attention required to hold the first representation in working memory.

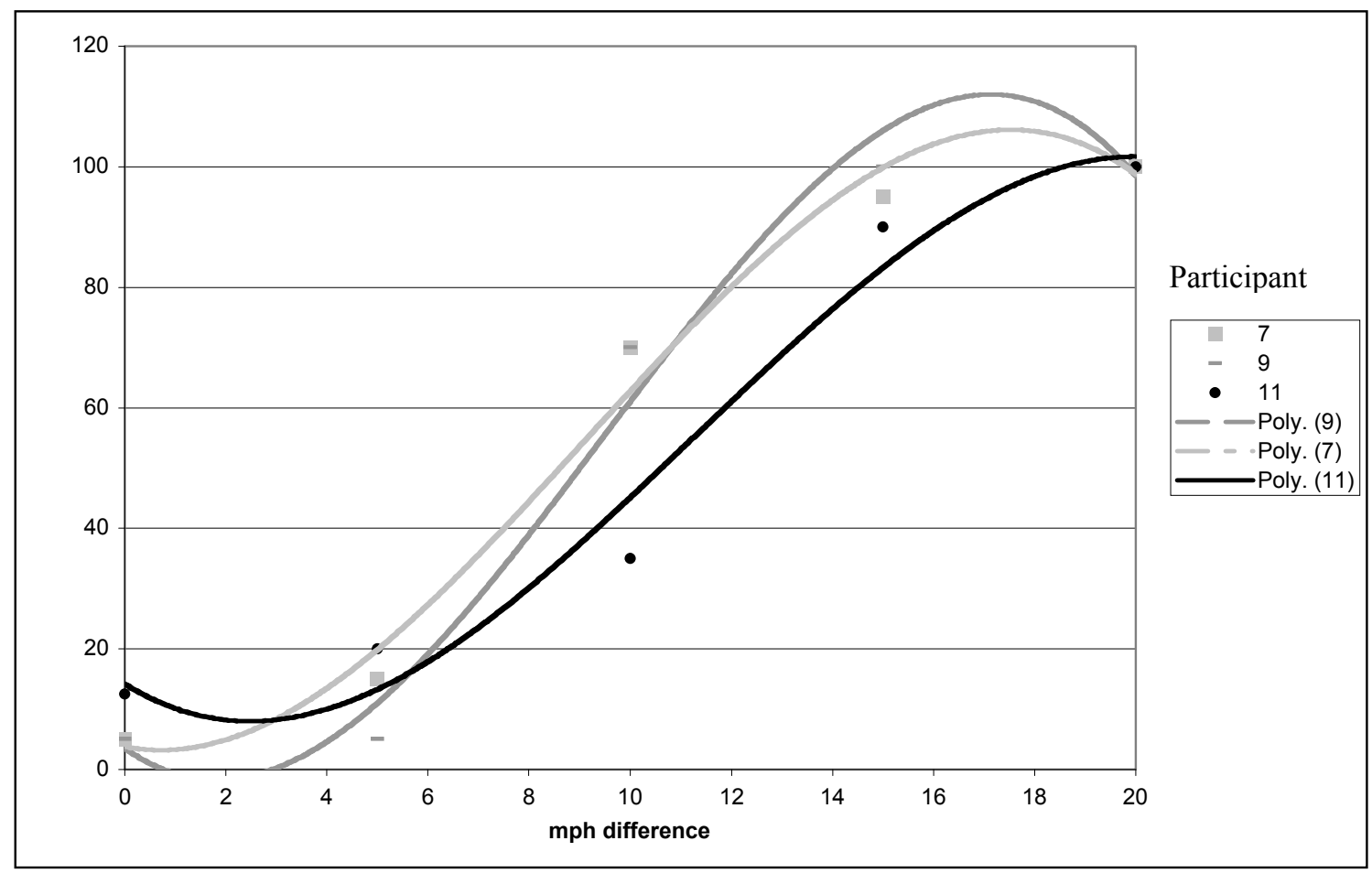

Figure 3. Sample of results from experiment 3

\section{REFERENCES}

Carrara 4 [Computer software]. (2005). Mountain View, CA: Eovia Corporation.

Corso, John F. (1967). The Experimental Psychology of Sensory Behavior. New York: Holt, Rinehart and Winston.

Inquisit 2.0.51002.1 [Computer software]. (2005). Seattle, WA: Millisecond Software LLC. 